\title{
Effect of dehydration on the pharmacokinetics of oxytetracycline hydrochloride administered intravenously in goats (Capra hircus)
}

\begin{abstract}
1. The effects of various levels of dehydration induced by water deprivation were studied in six Nubian goats on the pharmacokinetics of oxytetracycline after intravenous administration $(5 \mathrm{mg} / \mathrm{kg})$.

2. In goats that had lost an average of $7.6 \%$ body weight after 2 days of water deprivation, the elimination rate constant of the drug was significantly decreased $(\mathrm{P}<0.01)$ and the total body clearance was significantly slower $(\mathrm{P}<0.001)$. No statistically significant changes were observed in the pharmacokinetic parameters describing the distribution of the drug at this dehydration level.

3. Water deprivation for 3 or 4 days resulted in a level of dehydration at which the goats lost an average of $10.3 \%$ or $12.7 \%$ of their body weight, respectively; significant changes were observed in the pharmacokinetic distribution and elimination parameters of oxytetracycline. The volume of distribution at steady state was significantly decreased $(\mathrm{P}<0.01)$. Significantly slower total body clearance $(\mathrm{P}<0.001)$ and subsequent prolongation of the elimination halflife were found at these dehydration levels.

4. The alterations caused by dehydration on the disposition kinetics of the drug should be considered for better definition of dosage regimens for sick, dehydrated animals.
\end{abstract}

Keyword: Dehydration; Goat; Oxytetracycline; Pharmacokinetics 